\title{
Structure et géographie des réseaux d'innovation. Une analyse des processus d'innovation des entreprises de biotechnologies de Bâle et de Strasbourg
}

Structure and geography of innovation networks. A survey of innovation processes of biotechnology industries in Strasbourg and Basel. Räumliche Organisation der Innovationsnetzwerke. Eine Analyse der Innonvationsprozesse von Biotechnologieunternehmen aus Strasbourg und Basel.

\section{Christof Klöpper}

\section{(2) OpenEdition} Journals

Édition électronique

URL : http://journals.openedition.org/rge/1713

DOI : $10.4000 /$ rge. 1713

ISSN : 2108-6478

Éditeur

Association des géographes de l'Est

Édition imprimée

Date de publication : 1 septembre 2008

ISSN : 0035-3213

Référence électronique

Christof Klöpper, «Structure et géographie des réseaux d'innovation. Une analyse des processus d'innovation des entreprises de biotechnologies de Bâle et de Strasbourg », Revue Géographique de I'Est [En ligne], vol. 48 / 3-4 | 2008, mis en ligne le 24 mars 2010, consulté le 08 septembre 2020. URL : http://journals.openedition.org/rge/1713; DOI : https://doi.org/10.4000/rge.1713

Ce document a été généré automatiquement le 8 septembre 2020

Tous droits réservés 


\section{Structure et géographie des réseaux d'innovation. Une analyse des processus d'innovation des entreprises de biotechnologies de Bâle et de Strasbourg}

Structure and geography of innovation networks. A survey of innovation

processes of biotechnology industries in Strasbourg and Basel.

Räumliche Organisation der Innovationsnetzwerke. Eine Analyse der Innonvationsprozesse von Biotechnologieunternehmen aus Strasbourg und Basel.

\section{Christof Klöpper}

\section{Introduction}

1 L'innovation constitue un thème central de la recherche en sciences économiques. Dans ce cadre, il est généralement entendu qu'un grand nombre de facteurs influencent la capacité d'innovation des entreprises, et que l'innovation exerce une influence importante sur le succès des entreprises et sur le développement économique des régions et des pays. L'innovation constitue un processus interactif, dans lequel un grand nombre d'acteurs sont impliqués dans les réseaux d'innovation. Il est supposé que la proximité géographique entre les acteurs impliqués exerce une action positive sur les performances liées à l'innovation et que les clusters régionaux possèdent une grande importance pour les réseaux d'innovation. Dans le même temps, il est généralement reconnu que les réseaux d'innovation ne se limitent pas à l'échelle régionale d'un cluster mais s'étendent souvent à une échelle internationale. Jusqu'ici, la compréhension de l'organisation et de la géographie de ces réseaux d'innovation 
demeure partielle et des travaux sont nécessaires pour comprendre quels sont les acteurs impliqués et quelle est leur localisation, en particulier en ce qui concerne les transferts de savoir et de technologie. Cela s'explique par le fait que ces réseaux sont difficiles à appréhender et à représenter par les méthodes traditionnelles. Ce travail se propose d'y remédier sur la base d'une méthode bibliométrique, qui permet de représenter les réseaux d'innovation des entreprises de biotechnologies de Bâle et de Strasbourg, de les comparer et de les inscrire dans leur contexte économique régional.

\section{L'innovation et la géographie des réseaux d'innovation}

2 Que sait-on des processus d'innovation actifs dans une industrie high-tech comme les biotechnologies? La recherche relative aux processus d'innovation a montré que l'innovation diffère selon les contextes régionaux et nationaux. Des inputs similaires ne conduisent pas à des processus et à des outputs identiques. Les approches des systèmes régionaux d'innovation tentent de cerner la dépendance des processus d'innovation à leur contexte spécifique (Cooke et al. 1997, Asheim et Gertler 2005). Outre l'environnement scientifique et économique, le contexte politique et les réseaux régionaux liant les entreprises aux autres acteurs jouent un rôle central dans le déroulement des processus d'innovation (Tödtling et Trippl 2005).

3 Il est également aujourd'hui reconnu que les processus d'innovation ne suivent pas un cours linéaire dans les industries de haute technologie (Kline et Rosenberg 1986, Etzkowitz et Leydesdorf 2000). Cela signifie que les frontières entre les entreprises sont de plus en plus souvent dépassées dans les processus d'innovation. En outre, les grandes entreprises, qui avaient auparavant constitué leurs propres laboratoires de recherche, utilisent de plus en plus de ressources externes. Elles doivent être ouvertes à des technologies développées en dehors de l'entreprise et être capables de les intégrer (Cohen et Levinthal 1990). Cette évolution, également appelée open innovation, repose sur des réseaux d'acteurs différents et spécialisés (Chesbrough 2003, von Hippel 2005). $\mathrm{Au}$ sein de ces réseaux d'innovation, certaines jeunes entreprises technologiques et certains organismes de recherche publics gagnent en importance (Pavitt 1991, Dasgupta et David 1994, The Economist 2007).

4 Les changements relatifs à l'organisation des processus d'innovation ainsi que l'influence du contexte sur ces processus ont des conséquences sur la géographie des processus d'innovation. En effet, les partenaires potentiels des acteurs de l'innovation sont théoriquement disponibles à l'échelle mondiale et certaines études montrent que les entreprises font usage de cette possibilité, de façon à sélectionner les meilleures partenaires pour leurs projets relatifs à l'innovation (voir par exemple Archibugi et Lundvall 2001, Zeller 2004, Birch 2008). Cependant, les processus d'innovation ne naissent pas de n'importe quel endroit de la planète et s'appuient sur des réseaux régionaux de clusters reliées entre eux. Cette structure, que Gertler et Levitte (2005) décrivent comme des nœuds locaux dans des réseaux globaux (« local nodes in global networks ») en référence à la formule d'Amin et Thrift (1992) («neo-marshallian nodes in global networks »), réunit des tendances à la dispersion et à la concentration.

5 Les connaissances relatives à la géographie et à l'organisation précise des réseaux liés à l'innovation restent encore lacunaires. L'image qui est donnée des réseaux d'innovation 
demeure encore floue et plusieurs questions restent encore largement irrésolues: comment se combinent les relations régionales et internationales? A quelle fréquence les partenaires que sont les universités, les entreprises orientées vers la R\&D et les grandes entreprises multinationales s'associent-elles? Dans quelle mesure les clusters diffèrent-ils en termes de réseaux d'innovation? Telles sont les questions abordées par le présent article, qui présente une partie des résultats d'une vaste étude portant sur les réseaux d'innovation des entreprises de biotechnologies conduite de 2006 à 2008 à l'échelle de onze pays européens et de la Suisse (voir Klöpper sous presse). Dans cette étude, les clusters de Zurich, Genève, Lausanne, Berne, Munich, Rhin-Neckar, Paris et Île-de-France, Cambridge et Oxford ont été particulièrement étudiés. Ces exemples sont mobilisés dans cet article dans la mesure où ils contribuent à la compréhension des réseaux d'innovation de Bâle et de Strasbourg.

\section{L'innovation dans les biotechnologies}

6 Les biotechnologies forment un secteur jeune et stratégique, marqué par une forte dynamique scientifique et économique. Les domaines principaux des biotechnologies se rapportent au secteur pharmaceutique (aussi appelées red technology). Dans ce domaine, les biotechnologies n'ont pas seulement apporté un saut technologique, mais également d'importants changements économiques. Alors que les biotechnologies sont utilisées par presque l'ensemble des entreprises de l'industrie pharmaceutique (y compris les sociétés pharmaceutiques traditionnelles), l'avènement de ces technologies a contribué à donner naissance à un nouveau type d'entreprises. Plus petites et flexibles, ces entreprises occupent aujourd'hui une position stratégique dans la commercialisation des produits biotechnologiques et pharmaceutiques et sont considérées avec beaucoup d'espoir par les grands laboratoires pharmaceutiques, qui espèrent qu'elles pourront alimenter la commercialisation de leurs produits dans le futur (voir notamment Prevezer 2000, OCDE 2006 et 2007). Ces entreprises font l'objet de cette recherche.

7 Le déroulement et l'organisation des processus d'innovation en biotechnologie a reçu une attention considérable au cours des dernières années. Cela s'explique par le fait que les biotechnologies sont fortement liées à la connaissance fondamentale et représentent un idéaltype de la recherche scientifique (Cooke 2004). Les processus d'innovation dans l'industrie des biotechnologies sont étroitement liés à ceux de l'industrie pharmaceutique. La plupart des entreprises concernées développent de nouveaux médicaments ou de nouveaux diagnostics sur la base des biotechnologies. Les éléments suivants résument certains résultats obtenus par des études précédentes portant sur les processus d'innovation dans le domaine des biotechnologies :

8 L'industrie des biotechnologies présente une structure spatiale fortement concentrée sur quelques centres principaux appelés megacentres par Cooke (2004), nodes of excellence par Feldman (2001) ou clusters par Cortright et Maier (2002).

9 L'industrie des biotechnologies est fortement intégrée dans l'économie mondiale. Les entreprises concernées desservent les marchés mondiaux et forgent des alliances internationales destinées à la commercialisation de leurs produits (Powell et al. 1996, Lawton Smith et Bagchi-Sen 2004, Birch 2008). La structure exacte et l'échelle de ces interdépendances demeurent à ce jour encore mal connues.

Un cluster biotechnologique typique est généralement constitué autour des universités et des centres de recherche et se compose d'un grand nombre de petites entreprises 
orientées vers la R\&D, qui peuvent dans de nombreux cas être elles-mêmes issues des universités ou des centres de recherche (Feldman et Francis 2003, Kaufmann et al. 2003, Klöpper sous presse).

\section{Mesurer les réseaux d'innovation à l'aide de données bibliographiques}

11 L'analyse des réseaux constitue un problème central en sciences économiques et sociales. La méthode souvent utilisée qui consiste à reconstituer des réseaux par voie d'enquête ou d'entretiens présente de sérieux handicaps qui diminuent l'impact des données collectées. La subjectivité et les compétences des personnes enquêtées sont ainsi fortement susceptibles d'influencer les données récoltées. Dans le présent travail, les réseaux d'innovation sont appréhendés grâce aux données bibliométriques, c'est-àdire sur la base des publications des chercheurs travaillant dans les entreprises de biotechnologies. Ces publications scientifiques constituent des indicateurs des interactions au sein des réseaux d'innovation et les co-auteurs d'une publication sont considérés comme liés entre eux. Une telle utilisation de données bibliométriques pour mesurer les réseaux d'entreprises est encore nouvelle, mais offre de grands avantages par rapport aux méthodes traditionnelles mentionnées ci-dessus.

12 Les principaux avantages de cette méthode sont de présenter des résultats indépendants de l'opinion des personnes interrogées et de permettre des comparaisons entre différents réseaux au cours du temps. En outre, cette méthode est adaptée à la collecte de données issues de très grands réseaux d'innovation. Il existe cependant des limites à utiliser la méthode bibliométrique : cette méthode ne se prête qu'à la collecte d'informations relatives à des relations formelles et dans cet article seules les personnes ou les secteurs économiques qui rédigent des publications peuvent être considérées. C'est le cas dans les biotechnologies, car les entreprises concernées publient leurs résultats de recherche du fait qu'elles y sont astreintes légalement en vue de l'autorisation de commercialisation d'un médicament ou parce que le processus de publication constitue un moyen utile et gratuit permettant de bénéficier de conseils sur le développement des produits. L'interprétation des données bibliométriques comporte un biais scientifique dans le sens que la participation des partenaires y est surévaluée par rapport à la réalité. Cela s'explique par le fait que les publications scientifiques constituent un indicateur de performance, alors que dans l'industrie elles ne sont qu'un moyen de révision des résultats de la R\&D. Comme ce biais affecte toutes les entreprises et tous les clusters de la même manière, il ne limite pas les comparaisons effectuées à l'échelle des entreprises ou des régions. Une description détaillée ainsi qu'une critique de cette méthode se trouvent dans Klöpper (sous presse).

\section{Les clusters étudiés}

\section{A. Le cluster biotechnologique de Bâle et son environnement institutionnel}

Le cluster de biotechnologies bâlois comprend 34 entreprises, dont 27 sont représentées dans l'analyse des interactions qui suit (jusqu'en mai 2007). Bâle possède ainsi la plus grande concentration d'entreprises de biotechnologies de Suisse. Ce pays 
est aujourd'hui considéré comme l'un des centres principaux des biotechnologies dans le monde et présente la plus forte densité d'entreprises actives dans ce domaine par habitant (Ernst \& Young 2008). L'industrie des biotechnologies suisses est fortement décentralisée géographiquement et, outre Bâle, plusieurs autres clusters existent à Zurich, Genève, Lausanne et, dans une moindre mesure, à Berne (Klöpper sous presse).

La genèse du cluster bâlois est différente de celle d'autres clusters, dans la mesure où seulement $18,4 \%$ des entreprises de biotechnologies de Bâle sont issues des universités ou des instituts de recherche (la moyenne des onze cas d'étude précédemment évoqués est de $40,3 \%$ ). En revanche, $21,1 \%$ des entreprises de biotechnologies bâloises sont issues de l'industrie pharmaceutique, contre seulement $12,6 \%$ en moyenne dans les autres clusters. Le cluster bâlois se compose d'entreprises à la fois déjà bien établies et partiellement cotées en bourse, et également de petites et jeunes startups.

Bâle est l'un des centres mondiaux de l'industrie pharmaceutique. Cette industrie occupe une proportion sensiblement supérieure de l'emploi régional et deux des plus grandes entreprises pharmaceutiques du monde y ont leur siège social, Novartis et Roche (Schneider-Sliwa et al. 2001, Cooke 2005, OCDE 2007). En outre, Bâle possède une forte densité d'entreprises connexes au secteur pharmaceutique, comme des prestataires de services ou des fournisseurs. Contrairement à d'autres clusters biotechnologiques, Bâle ne possède pas un environnement académique particulièrement développé : les acteurs principaux du domaine scientifique sont certes spécialisés dans les sciences de la vie mais l'université et la haute école travaillant dans le domaine sont relativement petites.

16 A cela s'ajoute le fait que, contrairement à d'autres clusters connus dans le monde, la région de Bâle n'encourage pas particulièrement le développement des biotechnologies par une promotion spécifique (OCDE 2007, Klöpper sous presse). Outre la politique économique libérale bien connue de la Suisse, il faut également compter sur la forte fragmentation administrative de la région. La région de Bâle est en effet composée de plusieurs cantons et de plusieurs communes françaises et allemandes. Le cluster bâlois se concentre presque exclusivement sur la partie suisse de l'agglomération au détriment des parties situées en France et en Allemagne.

\section{B. Le cluster biotechnologique de Strasbourg et son contexte institutionnel}

17 La France ne figure pas au rang des principaux lieux de concentration des biotechnologies dans le monde (Reiss 2003, Avenel et al. 2005, Ernst \& Young 2005). En comparaison des Etats-Unis, de la Grande-Bretagne ou de la Suisse, la France compte en effet relativement peu d'entreprises de biotechnologies et seul un petit nombre d'entre elles possèdent des produits prêts à être commercialisés (médicaments ou diagnostics) ou des projets de recherche proches de la phase de commercialisation.

18 Contrairement à l'industrie suisse des biotechnologies, les entreprises françaises sont fortement concentrées géographiquement, surtout dans l'île-de-France (Corolleur et al. 2003). Après cette région, Strasbourg constitue l'un des principaux centres de l'industrie biotechnologique française. Contrairement à Bâle, Strasbourg possède un grand acteur central bien implanté historiquement, l'entreprise Transgene. Cette entreprise, fondée en 1981, compte parmi les pionniers de la commercialisation des biotechnologies en Europe. Outre Transgene, 13 autres entreprises de biotechnologies 
sont implantées à Strasbourg, dont la plupart sont de petites structures qui sont souvent également actives dans la fourniture de services. A l'opposé des entreprises bâloises, les firmes strasbourgeoises sont souvent issues des universités ou des centres de recherche, notamment de l'Université Louis Pasteur.

L'économie strasbourgeoise n'est pas, contrairement à celle de Bâle, fortement spécialisée sur l'industrie chimique et pharmaceutique et cela malgré la présence des entreprises Eli-Lily et Sanofi-Aventis (selon la valeur ajoutée et le nombre d'employés calculés par BAK Basel 2007). En revanche, Strasbourg est caractérisée par une haute densité d'infrastructures de recherche dans le domaine. Le cœur de la recherche en biotechnologies est l'Ecole Supérieure de Biotechnologie de Strasbourg (ESBS) de l'Université Louis Pasteur et le Parc d'innovation d'Illkrich. En tant que Pôle de compétitivité dans le domaine des biotechnologies et de la pharmacie, la recherche et l'économie bénéficient de financements attribués par le gouvernement central de Paris. De ce fait, les entreprises strasbourgeoises possèdent nettement plus de possibilités de promouvoir leurs recherches comparativement à la Suisse.

\section{Comparaison des réseaux d'innovation}

20 Cette contribution analyse trois dimensions des réseaux d'innovation : l'organisation des réseaux et des collaborations entre entreprises; la géographie des réseaux, en particulier les interactions internes et externes aux clusters; la structure interne des relations.

Ce travail couvre un total de 357 interactions ayant eu lieu entre les entreprises de biotechnologies de Bâle et leurs 546 partenaires depuis 1990, ainsi qu'un total de 738 interactions ayant eu lieu entre les entreprises de Strasbourg et leurs 1425 partenaires. Les onze autres cas étudiés comptent quant à eux 5442 interactions pour 10658 partenaires (Tableau 1). Le nombre élevé d'interactions relatives à Strasbourg est presque exclusivement dû à la R\&D conduite par l'entreprise Transgene, qui occupe une place centrale dans le cluster strasbourgeois (voir chapitre V. B.).

\section{A. Organisation des réseaux d'innovation}

Les réseaux d'innovation des entreprises étudiées lient les différents acteurs de la chaîne de valeur de l'industrie biotechnologique et pharmaceutique. Ces acteurs sont par exemple les universités, les hôpitaux universitaires, les prestataires spécialisés, les entreprises chimiques et pharmaceutiques ou d'autres entreprises biotechnologiques. La présente analyse montre que les relations dans les différents clusters présentent clairement des différences et notamment en ce qui concerne l'importance des partenaires scientifiques et économiques ${ }^{1}$. Alors qu'à Bâle les entreprises sont plus orientées vers le marché, les entreprises strasbourgeoises sont quant à elles plus orientées vers le monde scientifique (voir Tableau 1). Cette orientation des firmes strasbourgeoises, typique des entreprises de biotechnologies françaises en général, est surtout due au fait que les entreprises entretiennent une collaboration plus étroite avec les partenaires de la recherche publique qu'avec d'autres entreprises situées dans d'autres clusters. Ceci est une conséquence du système d'innovation français, qui compte une grande variété d'organismes publics de recherche tels que le CNRS ou l'INSERM par exemple, ainsi que de nombreux financements publics destinés au 
développement des activités de R\&D. L’orientation économique de Bâle constitue une particularité, qui se distingue également des onze autres cas considérés et n'est en aucun cas typique des autres clusters suisses. Cet aspect s'ajoute à la forte proportion d'entreprises issues de l'industrie pharmaceutique liée à l'histoire particulière du cluster bâlois et permet de qualifier ce cas d'étude de corporate innovation system, un système d'innovation régional particulièrement orienté sur la sphère économique et le marché.

Tableau 1. Entreprises de biotechnologies et réseaux d'innovation

\begin{tabular}{|l|l|l|l|l|l|}
\hline & $\begin{array}{l}\text { Nombre } \\
\text { d'entreprises }\end{array}$ & $\begin{array}{l}\text { Nombre } \\
\text { d'interactions }\end{array}$ & $\begin{array}{l}\text { Nombre de } \\
\text { partenaires }\end{array}$ & $\begin{array}{l}\text { Proportion de } \\
\text { partenaires } \\
\text { scientifiques, } \\
\text { en \%* }\end{array}$ & $\begin{array}{l}\text { Proportion de } \\
\text { partenaires } \\
\text { économiques, } \\
\text { en \%* }\end{array}$ \\
\hline Bâle & 34 & 357 & 546 & 76,55 & 23,06 \\
\hline Strasbourg & 13 & 738 & $1^{\prime} 425$ & 92,00 & 7,23 \\
\hline $\begin{array}{l}\text { Total des } \\
\text { autres cas } \\
\text { étudiés** }\end{array}$ & 288 & $5^{\prime} 442$ & $10^{\prime} 658$ & 87,69 & 11,71 \\
\hline
\end{tabular}

Source : calculs de l'auteur à partir du Web of Science 2007.

* La somme des proportions des partenariats scientifiques et économiques n'est pas équivalente à $100 \%$ du fait que certains partenaires n'ont pu être classés dans l'une ou l'autre des catégories. ** Y compris les entreprises étudiées dans les onze autres clusters.

\section{B. Géographie des réseaux d'innovation}

L'analyse de la géographie des réseaux d'innovation bâlois et strasbourgeois relatifs aux biotechnologies montre tout d'abord l'importance des interactions interrégionales pour ces entreprises: dans les deux clusters, plus de $80 \%$ des interactions s'adressent à des partenaires extérieurs à la région (voir Tableau 2). Ces résultats indiquent que les interactions régionales des clusters, particulièrement mises en évidence dans la littérature, ont relativement peu d'importance du point de vue quantitatif. Toutefois, il existe également certaines différences significatives entre les deux clusters étudiés : alors que les entreprises bâloises collaborent fréquemment avec des partenaires étrangers $(69,1 \%)$, les entreprises strasbourgeoises collaborent plus volontiers avec d'autres entreprises situées en France (42,1\%), et surtout avec celles situées en Île-deFrance $(26,9 \%$, soit plus de la moitié des interactions avec le reste de la France). Plusieurs raisons permettent de justifier cette différence. Premièrement, il est bien évident que plus un pays est petit et plus les réseaux d'innovation auront tendance à être internationaux (voir Klöpper sous presse). Deuxièmement, les facteurs formels et informels liés aux espaces linguistiques et aux marchés du travail exercent également une influence sur les réseaux d'innovation. Les entreprises bâloises travaillent intensivement avec des partenaires allemands par exemple. Alors que les coûts de transaction avec des partenaires situés dans une autre région linguistique devraient être faibles du fait que la langue de travail de nombreuses entreprises est l'anglais, il est 
probable que les liens privilégiés tissés entre le marché de l'emploi suisse alémanique et allemand jouent un rôle important. Ainsi, au cours des carrières, les contacts noués dans l'entreprise permettent par la suite de mettre en œuvre des projets de coopération au sein de réseaux d'innovation. Troisièmement, les systèmes universitaires et les politiques de la recherche jouent également un rôle. Ainsi, les organismes de promotion de la recherche nationaux exigent souvent la participation de plusieurs institutions à des projets de recherche. La proportion élevée d'interactions relevée pour les entreprises de Strasbourg peut également s'expliquer par la politique de promotion de la recherche et de l'économie française.

Le Tableau 2 montre également que l'origine des partenaires économiques et scientifiques diffère entre les deux clusters. A Bâle, les partenaires économiques proviennent à plus de $40 \%$ de la région, tandis qu'à Strasbourg, ce pourcentage est de moins de $1 \%$, une proportion qui correspond également à celle relevée dans la plupart des onze autres cas étudiés en Europe. Ceci s'explique d'une part par la disponibilité des partenaires existants dans l'industrie pharmaceutique ou chez les prestataires de services de Bâle, qui n'existe ni à Strasbourg ni dans la plupart des autres clusters étudiés. D'autre part, la forte proportion bâloise s'explique par les spécificités historiques des entreprises de cette ville, qui sont le plus souvent issues d'autres entreprises plutôt que des universités et qui ont le plus souvent gardé des contacts avec leurs firmes mères. Ceci montre que les réseaux d'innovation sont étroitement liés au développement des entreprises et à l'évolution des clusters.

Tableau 2. La géographie des réseaux d'innovation. Proportions des interactions entretenues avec les différents partenaires, en \%

\begin{tabular}{|l|l|l|l|l|l|l|}
\hline & \multicolumn{3}{|l|}{$\begin{array}{l}\text { Entreprises de Bâle } \\
\mathrm{n}=546\end{array}$} & $\begin{array}{l}\text { Entreprises de Strasbourg } \\
\mathrm{n}=1425\end{array}$ \\
\hline & $\begin{array}{l}\text { A l'échelle } \\
\text { régionale } \\
\text { l'échelle } \\
\text { nationale }\end{array}$ & $\begin{array}{l}\text { Avec } \\
\text { l'étranger }\end{array}$ & $\begin{array}{l}\text { A l'échelle } \\
\text { régionale }\end{array}$ & $\begin{array}{l}\text { A } \\
\text { l'échelle } \\
\text { nationale }\end{array}$ & $\begin{array}{l}\text { Avec } \\
\text { l'étranger }\end{array}$ \\
\hline $\begin{array}{l}\text { Milieu } \\
\text { scientifique }\end{array}$ & 11,48 & 15,31 & 73,21 & 18,38 & 41,88 & 39,74 \\
\hline $\begin{array}{l}\text { Milieu } \\
\text { économique }\end{array}$ & 42,86 & 1,59 & 55,56 & 0,97 & 41,75 & 57,28 \\
\hline \hline Total & 18,86 & 12,09 & 69,05 & 17,05 & 42,11 & 40,84 \\
\hline
\end{tabular}

Source : calculs de l'auteur à partir du Web of Science 2007.

\section{Analyse des réseaux internes aux clusters}

Les paramètres statistiques des réseaux internes aux clusters ont été calculés au moyen du logiciel Ucinet (Borgatti et al. 2002) et dessinés avec le logiciel Netdraw (voir Figures 1 et 2). La situation de chaque réseau dans la représentation graphique a été calculée sur la base de l'algorithme Spring-Embedding ${ }^{2}$. La position des acteurs calculée avec cet 
algorithme est sans rapport avec sa localisation géographique effective, mais résulte de sa position au sein des nœuds du réseau. Les réseaux d'innovation analysés sont égocentrés (les entreprises de biotechnologies étant considérées comme ego), ce qui signifie qu'ils sont examinés du point de vue des entreprises concernées. L'épaisseur des liens est proportionnelle à la fréquence des contacts entre les nœuds.

Les Figures 1 et 2 montrent que les réseaux possèdent des structures internes différentes. Le réseau d'innovation bâlois se distingue par sa structure décentralisée et polycentrique, ainsi que par l'importance des entreprises pharmaceutiques. Ainsi, les entreprises de biotechnologies de Bâle travaillent plus fréquemment avec les entreprises pharmaceutiques comme Roche qu'avec l'Université de Bâle ou l'hôpital universitaire (voir Figure 1). Il est difficile de délimiter des sous-parties à l'intérieur de ce réseau. L'étroite collaboration entretenue entre Novartis et l'Institut Friedrich Miescher (FMI) permet d'identifier un petit sous-groupe. De même, l'Université et l'hôpital universitaire d'un côté, et Novartis et Roche d'un autre côté forment deux autres regroupements d'entreprises, qui entretiennent cependant des liens avec d'autres partenaires. Le cluster bâlois est reconnaissable à sa forte densité de relations entre les entreprises de biotechnologies et d'autres acteurs, à l'opposé du cluster de Strasbourg dans lequel peu de passerelles existent entre les entreprises concernées, et par le faible nombre d'entreprises reliées à un large éventail de partenaires (voir Figure 2). Le principal acteur du réseau d'innovation est de loin l'Université de Strasbourg, responsable de $42 \%$ des interactions et qui travaille avec presque toutes les entreprises du secteur étudié. En raison de l'importance de cette université, le réseau strasbourgeois est fortement centralisé. Le second acteur important du réseau est l'entreprise Transgene, qui entretient certes des relations intenses avec différents organismes de recherche de Strasbourg mais qui n'est pas reliée à d'autres entreprises du même genre qu'elle. L'analyse des onze autres clusters de biotechnologies effectuée dans un cadre plus large indique qu'une structure centralisée telle que celle de Strasbourg est typique d'un petit cluster disposant de peu de partenaires potentiels. Les plus grands clusters se caractérisent en revanche en général par des réseaux décentralisés (Klöpper sous presse). 
Figure 1. Le réseau d'innovation régional de Bâle

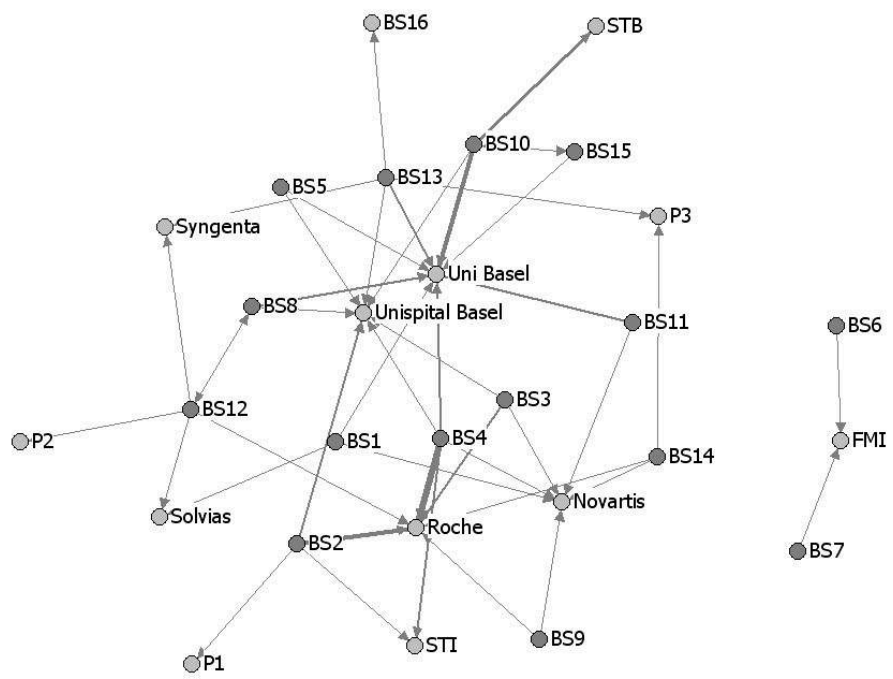

Outre l'Université de Bâle, les acteurs centraux du réseau d'innovation régional de Bâle sont les entreprises pharmaceutiques. L'Institut Friedrich Miescher constitue un petit réseau spécifique. La situation des nœuds isolés a été traitée à l'aide de l'algorithme Spring-Embedding et n'a pas de rapport avec la localisation géographique réelle des acteurs. $N=98$. Source : calculs de l'auteur à partir de Web of Science 2007. Les abréviations utilisées sont les suivantes: BS\#\# pour les entreprises biotechnologiques de Bâle, P\#\# pour les petites et moyennes entreprises de Bâle, FMI pour l'Institut Friedrich Miescher, STI pour l'Institut tropical suisse (Schweizerisches Tropeninstitut), STB pour la Banque de données sur les tumeurs de Bâle (Stiftung Tumorbank Basel). 
Figure 2. Le réseau d'innovation régional de Strasbourg

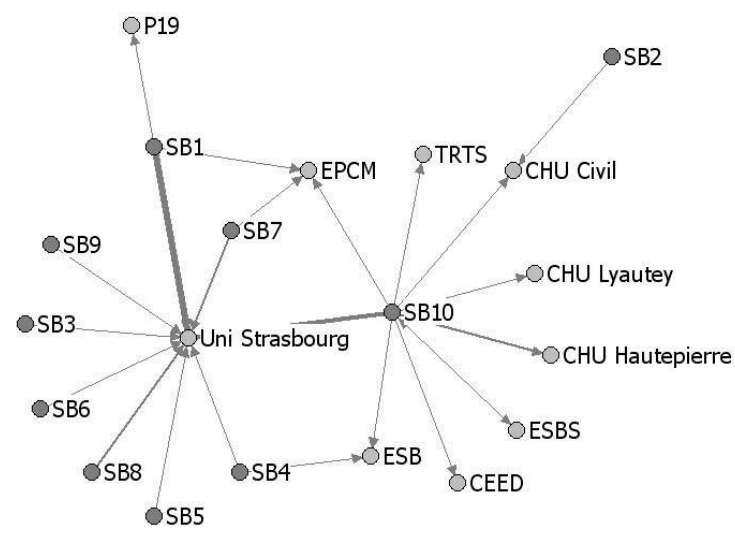

Outre l'Université de Strasbourg, l'entreprise de biotechnologie Transgene occupe une place centrale dans les réseaux d'innovation régionaux. $N=136$. Source : calculs de l'auteur à partir de Web of Science 2007. Pour une explication de la manière dont les réseaux ont été calculés, voir Figure 1. Les abréviations utilisées sont les suivantes : SB\#\# pour les entreprises biotechnologiques de Strasbourg, P\#\# pour les petites et moyennes entreprises de Strasbourg, CEED pour le Centre européen d'études du diabète, $\mathrm{CHU}$ pour le Centre hospitalier universitaire, EFSA pour l'Etablissement français du sang Alsace, ESB pour l'Ecole supérieure de biotechnologie de Strasbourg, EPCM pour l'Ecole européenne de chimie, polymères et matériaux de Strasbourg.

\section{Conclusion}

Les réseaux d'innovation étudiés présentent plusieurs différences sensibles, qui concernent la taille et la structure interne des réseaux formant les clusters, la proportion d'interactions entretenues avec des partenaires situés à l'échelle régionale, nationale ou internationale, ainsi que les acteurs concernés au sein des réseaux. Alors que les entreprises de biotechnologies de Bâle sont orientées vers le marché et l'échelle internationale, celles de Strasbourg sont plutôt orientées vers le milieu des sciences et l'échelle nationale, notamment l'île-de-France. Les entreprises de biotechnologies de Bâle font partie d'un réseau interne dense qui est né autour de l'industrie pharmaceutique et qui est peu intégré au système d'innovation suisse. A Strasbourg, le réseau d'innovation est sensiblement moins relié à l'Université de Strasbourg qu'avec le reste du pays.

La présente étude montre ainsi que la proximité géographique peut avoir des effets très différents selon les clusters considérés. A Bâle, les effets du cluster sont sans doute positifs, sous la forme d'une orientation plus économique liée à la proximité avec l'industrie pharmaceutique. Il est probable que cette orientation économique des processus d'innovation et des réseaux ait contribué à la réussite du cluster bâlois. A 
Strasbourg, où il y a peu de liens avec l'industrie pharmaceutique et où les entreprises concernées ne collaborent pas fréquemment dans le domaine des biotechnologies, les effets du cluster sont sans doute plus faibles et n'influencent que modérément la réussite des entreprises en termes d'innovation.

Plusieurs facteurs peuvent expliquer ces différences. En premier lieu, la taille du cluster est importante : plus un cluster est grand et plus il existe de partenaires potentiels, plus les interactions régionales seront nombreuses, et plus la densité du réseau interne du cluster sera grande. En second lieu, l'histoire des entreprises et l'évolution du cluster jouent un rôle. Ceci est particulièrement évident à Bâle où les entreprises étudiées entretiennent des liens privilégiés avec l'industrie pharmaceutique qui s'y est développée depuis longtemps. En troisième lieu, les résultats de cette étude montrent que l'environnement institutionnel marque les réseaux d'innovation. Ainsi, la géographie des marchés de l'emploi, des aires linguistiques ainsi que les politiques économiques et de promotion de la recherche exercent une influence sur les réseaux étudiés.

Quelle est la signification de ces résultats en matière de recherche et de politique économique ou technologique? Tout d'abord, cette étude montre que les méthodes bibliométriques permettent d'apporter de nouveaux résultats empiriques dans le domaine des clusters, des réseaux d'innovation et des systèmes d'innovation, sur une base quantitative vérifiable. Ensuite, cette étude montre qu'il n'existe pas un seul type de cluster ou de système d'innovation régional, mais une diversité de cas. Les régions étudiées diffèrent selon leurs structures et le contexte dans lequel se développe l'innovation. Ces différences locales ont des effets sur les stratégies des entreprises et sur les réseaux d'innovation. Enfin, cette étude montre que des politiques de développement économiques adaptées aux différences observées entre les clusters devraient être appliquées. Ainsi, la promotion des biotechnologies à Bâle devrait s'appuyer sur les forces existantes dans la région, en tenant compte du fait que l'industrie des biotechnologies est déjà fortement liée à l'industrie pharmaceutique. Dans le cas des entreprises de biotechnologies de Strasbourg, les atouts existants dans le milieu universitaire local pourraient constituer le point de départ d'une promotion adéquate des biotechnologies au niveau régional.

\section{BIBLIOGRAPHIE}

Amin, A., Thrift, N.J. (1992). - « Neo-Marshallian nodes in global networks », International Journal of Urban and Regional Research, $\mathrm{n}^{\circ} 16, \mathrm{p}$. 571-587.

Archibugi, D., Lundvall, B.Å. (éds) (2001). - The globalizing learning economy, Oxford.

Asheim, B.T., Gertler, M. (2005). - « Regional innovation systems and the geographical foundations of innovation », in Fagerberg, J., Mowery, D., Nelson, R. (éds) : The Oxford handbook of innovation, Oxford, p. 291-317. 
Avenel, E., Corolleur, F., Gauthier, C., Rieu, C. (2005). - Start-ups, firm growth and the consolidation of the French biotech industry, Working Papers du Laboratoire d'Economie Appliquée de Grenoble, Grenoble.

Birch, K. (2008). - « Alliance-driven governance : Applying a global commodity chains approach to the UK biotechnology industry ", Economic Geography, n 84/1, p. 83-103.

Borgatti, S.P., Everett, M.G., Freeman, L.C. (2002). - Ucinet for Windows : Software for social network analysis, Cambridge, MA.

Chesbrough, H.W. (2003). - Open innovation. The new imperative for creating and profiting from technology, Boston.

Cohen, W.M., Levinthal, D.A. (1990). - « Absorptive capacity : A new perspective on learning and innovation ", Administrative Science Quarterly, n 35/1, p. 128-152.

Cooke, P. (2004). - « The molecular biology revolution and the rise of bioscience megacentres in North America and Europe », Environment and Planning C : Government and Policy, $\mathrm{n}^{\circ} 22 / 2$, p. 161-177.

Cooke, P., Uranga, M., Gomez Etxebarria, G. (1997). - « Regional innovation systems : Institutional and organisational dimensions », Research Policy, $n^{\circ} 26$, p. 475-491.

Cooke, P. (2005). - « Regionally asymmetric knowledge capabilities and open innovation. Exploring « globalisation 2 »-A new model of industry organisation », Research Policy, $\mathrm{n}^{\circ} 34$, p. 1128-1149.

Corolleur, F., Mangematin, F., Torre, A., Lemaire, S. (2003). - « French biotech start-ups and biotech clusters in France: The importance of geographic proximity ", in Fuchs, G. (éds). Biotechnology in comparative perspective, Londres, New York, p. 98-124.

Cortright, J., Mayer, H. (2002). - Signs of life: The growth of biotechnology centers in the U.S, Washington, DC.

Dasgupta, P., David, P.A. (1994). - « Towards a new economics of science », Research Policy, $\mathrm{n}^{\circ} 23$, p. 487-521.

Ernst \& Young (2005). - Beyond Borders. Global Biotechnology Report 2005.

Ernst \& Young (2008). - Swiss biotech report, Bâle.

Etzkowitz, H., Leydesdorf, L. (2000). - « The dynamics of innovation: From national innovation systems and " mode 2 » to a triple helix of university-industry-government relations ",.Research Policy, $\mathrm{n}^{\circ} 29$, p. 109-123.

Fagerberg, J., Mowery, D., Nelson, R. (éds) (2005). - The Oxford handbook of innovation, Oxford.

Feldman, M.P. (2001). - « The entrepreneurial event revisited : An examination of new firm formation in the regional context ", Industrial and Corporate Change, $n^{\circ} 10$, p. 861-891.

Feldman, M.P., Francis, J.L. (2003). - « Fortune favours the prepared region: The case of entrepreneurship and the capitol region biotechnology cluster », European Planning Studies, $n^{\circ} 11 / 7$, p. $765-788$.

Fuchs, G. (éd.) (2003). - Biotechnology in comparative perspective, Londres, New York.

Gertler, M., Levitte, Y.M. (2005). - « Local nodes in global networks: The geography of knowledge flows in biotechnology innovation ", Industry and Innovation, $n^{\circ} 12$, p. 487-507. 
Kaufmann, D., Schwartz, D., Frenkel, A., Shefer, D. (2003). - « The role of location and regional networks for biotechnology firms in Israel », European Planning Studies, n 11/7, p. 823-840.

Kline, S.J., Rosenberg, N. (1986). - « An overview of innovation », in Landau, R., Rosenberg, N. (éds). - The positive sum strategy : Harnessing technology for economic growth, Washington, p. 275-307.

Klöpper, C. (sous presse). - Globale Netzwerke, regionale Einflüsse : Die Struktur und Geographie von Innovationsprozessen in der Biotechnologie. Eine Analyse der Innovationsnetzwerke von Biotechnologieunternehmen in elf Clustern in der Schweiz, Deutschland, Frankreich und Grossbritannien.

Landau, R., Rosenberg, N. (éds) (1986). - The positive sum strategy: Harnessing technology for economic growth, Washington.

Lawton-Smith, H., Bagchi-Sen, S. (2004). - « Innovation geographies : International perspectives on research, product development, and commercialisation of biotechnologies ", Environment and Planning C : Government and Policy, $\mathrm{n}^{\circ} 22 / 2$, p. 159-160.

OCDE (2006). - Innovation in pharmaceutical biotechnology. Comparing national innovation systems at the sectoral level, Paris.

OCDE (2007). - Globalisation and regional economies - Case studies in the biotechnology sector, Paris.

Pavitt, K. (1991). - « What makes basic research economically useful ?», Research Policy, $\mathrm{n}^{\circ}$ 20/2, p. 109-119.

Powell, W.W., Koput, K.W., Smith-Doerr, L. (1996). - « Interorganizational collaboration and the locus of innovation : Networks of learning in biotechnology ", Administrative Science Quarterly, $\mathrm{n}^{\circ} 41$, p. $116-145$.

Prevezer, M. (2000). - « Ingredients in the early development of U.S. biotechnology industry », Small Business Economics, $\mathrm{n}^{\circ}$ 17, p. 17-29.

Reiss, T. (2003). - Efficiency of innovation policies in high technology sectors in Europe, Luxembourg.

Schneider-Sliwa, R., Kampschulte, A., Sandtner, M., Strassmann, R., Volman, R. (2001). Wirtschaftsstruktur und Wirtschaftsdynamik der Nordwestschweiz, Bâle, Office statistique du canton de Bâle-Ville : Stadt und Region 2.

The Economist (2007). - The rise and fall of corporate R\&D. Out of the dusty labs.

Tödtling, F., Trippl, M. (2005). - « One size fits all ? Towards a differentiated regional innovation policy approach », Research Policy, n 34, p. 1203-1219.

von Hippel, E. (2005). - Democratizing innovation, Boston, MA, Londres.

Zeller, C. (2004). - « North-Atlantic innovative relations of Swiss pharmaceuticals and the importance of regional biotech arenas », Economic Geography, nº 80/1, p. 83-111.

\section{NOTES}

1. Le taux élevé de partenaires scientifiques est la conséquence de la méthode utilisée (et de son biais) et le contenu ne peut donc pas être interprété.

2. Le Spring Embedding permet d'attirer les nœuds reliés entre eux par analogie avec un modèle gravitaire alors que les nœuds non reliés se repoussent. L'attraction et la répulsion sont calculées en fonction des itérations successives en calculant à chaque itération les forces agissant sur chaque nœud. Après un nombre suffisamment grand d'itérations, un état d'équilibre est atteint, 
dans lequel la force exercée sur chaque nœud = 0. Dans cet article, l'algorithme Spring Embedding est la méthode qui a permis de représenter le plus clairement les réseaux.

\section{RÉSUMÉS}

Cet article étudie les réseaux d'innovation des entreprises de biotechnologies de Bâle et de Strasbourg au moyen d'une méthode bibliométrique qui permet de les comparer et de les situer dans leur contexte régional. Les résultats montrent que les réseaux d'innovation diffèrent significativement du point de vue de leur structure et de leur géographie. Ces différences peuvent être expliquées notamment par la taille des clusters biotechnologiques, par l'historique des entreprises et par l'environnement institutionnel.

This article presents results of a survey of innovation networks of biotechnology industries in Strasbourg and Basel using a bibliometric approach. The data collected was compared and interpreted according to regional contexts. The survey shows that the innovation networks in this region differ significantly in terms of organisation and geography. These differences can be explained by the varying sizes, development and institutional environments of investigated biotechnological clusters.

Für den vorliegenden Beitrag wurden Innovationsnetzwerke von Biotechnologieunternehmen aus Strasbourg und Basel mittel bibliometrischer Verfahren gemessen, miteinander verglichen und in ihren regionalen Kontext gestellt. Die Ergebnisse zeigen, dass sich die Innovationsnetzwerke erheblich bezüglich ihrer Organisation und ihrer Geographie unterscheiden. Diese Unterschiede lassen sich unter anderem durch die Grösse der untersuchten Biotechnologiecluster, durch die Entstehung der Unternehmen und durch das institutionelle Umfeld erklären.

\section{INDEX}

Keywords : Basel, biotechnology, cluster, innovation, innovation networks, Strasbourg Schlüsselwörter : Basel, Biotechnologie, Cluster, Innovation, Innovationsnetzwerke, Strasbourg Mots-clés : Bâle, biotechnologies, cluster, innovation, réseaux d'innovation, Strasbourg

\section{AUTEUR}

\section{CHRISTOF KLÖPPER}

Amt für Wirtschaft und Arbeit Basel-Stadt - Utengasse 36 Postfach 4005 Basel christof.kloepper@bs.ch 